\title{
Mujeres premiadas en concursos de letras flamencas
}

\author{
Domingo Giménez Cánovas \\ Catedrático jubilado de Informática de la Universidad de Murcia \\ Autor de letras flamencas
}

\begin{abstract}
En el presente trabajo se analiza la participación de mujeres en concursos de letras flamencas, los premios y menciones conseguidos así como la evolución de la participación femenina en dichos eventos.
\end{abstract}

Palabras clave: Letras flamencas; mujeres letristas; premios de letras flamencas.

This paper analyzes the participation of women in flamenco literary competitions, the awards and mentions achieved, as well as the evolution of female participation in these events.

\section{Introducción}

$\mathrm{Al}$ igual que en muchos otros campos, la mujer está infrarrepresentada en algunas facetas del flamenco. Por ejemplo, estamos trabajando en un "Inventario de premios de letras flamencas", y de los 338 letristas localizados que han resultado premiados en estos concursos, solo 57 de ellos son mujeres, lo que representa un $16.9 \%$. Es un porcentaje realmente bajo, que se sitúa a nivel del de los estudios con más baja representación de mujeres, alrededor de $13 \%$ en los de Informática y del $28 \%$ en otros estudios de Ingeniería 1

Volviendo al flamenco, la presencia de la mujer es también minoritaria en disciplinas como la guitarra. En Wikipedia no encontramos ninguna entrada de mujeres guitarristas flamencas, mientras que hay 79 de hombres, y en el blog El Arte de Vivir el Flamenco ${ }^{2}$ el porcentaje de mujeres tocaoras respecto del total solo alcanza un $9 \%$. En cambio, en el baile la situación es diferente pues, según los datos que encontramos en dichos sitios web, las mujeres representan respectivamente el $67 \%$ y el $72 \%$. Pero en el cante, una vez más, baja ostensiblemente el porcentaje de representación femenina, respectivamente, con un $22 \%$ y un $23 \%$.

Por cierto que ni Wikipedia ni el blog "El Arte de Vivir el Flamenco" incluyen apartado alguno relativo a letristas (ya sean masculinos o femeninos), con lo que, a nuestro juicio, queda también patente la importancia secundaria que se concede a los autores de letras flamencas.

Miguel Alarcón (2004) dedica un apartado a analizar la presencia de la mujer en el concurso de letras "Mirando a la Torre" hasta 2002, y sus datos van en la línea de lo que señalamos. La única mujer galardonada hasta esa fecha ha sido Carmen Aguirre, que nunca obtuvo el primer premio; sí un segundo, en nueve ediciones, y tres terceros premios, lo que representa porcentajes de $0 \%$, $11.1 \%$ y $33.3 \%$, y un porcentaje de $25 \%$ si consideramos todos los premios. Señala Alarcón que la participación femenina en el concurso de baile es del $80 \%$, con un porcentaje de ganadoras del $50 \%$, mientras que la participación femenina en guitarra es nula. A partir de 2003 se registra un aumento de la participación femenina en el concurso de letras, obteniendo respectivamente el primer y segundo premio en dicho año Marisa Ferrer y Pilar Bugella.

\footnotetext{
${ }^{1}$ Recuperado de https://cutt.ly/aYoOMbL

${ }^{2}$ Recuperado de https://elartedevivirelflamenco.com/
}

Revista De Investigación sobre Flamenco "La madrugá" 
(C) Servicio de Publicaciones de la Universidad de Murcia

http://revistas.um.es/flamenco

En este trabajo analizamos la participación femenina en concursos de letras flamencas, a través de los premios y menciones recibidas, y estudiando la evolución de su participación en ellos.

Empezaremos con una sección en la que se enumeran los premios de letras flamencas que hemos sido capaces de localizar, y se señala en cuáles de ellos encontramos premios y menciones a mujeres letristas. A continuación, en la parte central de este trabajo, se indican los premios y menciones concedidos a mujeres y se analiza la evolución de los premios por ellas recibidos. Terminamos con un resumen de conclusiones y propuesta de trabajos futuros.

\section{Resumen de premios de letras flamencas}

Hemos conseguido información de 58 premios de letras flamencas. Los enumeramos a continuación indicando en cuáles de ellos encontramos representación femenina (resaltamos el nombre de estos premios) en los premios y menciones (especiales, honoríficas, como finalista, etc.). Por cada concurso damos el número de premios que hemos localizado como concedidos a hombres y a mujeres (h-m).

Cuando aparece (0-0) es porque no tenemos información de los premios en ninguna edición. A los concursos en los que encontramos participación de mujeres se les asigna un códigq ${ }^{3}$ que utilizaremos en las tablas que se incluyen en el artículd 4 . Agrupamos los concursos por provincias:

- En Almería, tenemos el Concurso de Letras de Saetas de la Peña El Morato, celebrado entre 1998 y $2004(5-0)$.

- En Cáceres tenemos:

- El Premio Caja Duero a letra de saetas en 1999 (1-0).

- El Certamen de saetas Señor de la Pasión en 2001 (1-0).

- El Concurso de saetas Teresa la Naveta, con premio de letras en 2017 (1-0).

- En Cádiz:

- El Concurso de Cante por Alegrías, en el que en algunas ediciones de concede premio a mejor nueva letra interpretada. En este tipo de concursos, cuando se premia un cantaor/ora, normalmente no sabemos si es el letrista (2-0).

- El Concurso de Cante por Peteneras de Paterna de Rivera (PePR) se celebra desde 1972, y algunas ediciones se concede un premio a mejor nueva letra interpretada (13-2).

- El Concurso de Cante de Mirabrás (MiCa) del que encontramos algunas referencias, aunque puede que coincida con el de cantes de Sanlúcar. Se conceden premios a nuevas letras $(1-1)$.

- El Concurso de Cantes de Sanlúcar (LeSL) con una larga tradición pero del que solo conocemos premios a nuevas letras unos pocos años (0-2).

- Concurso de letras de saetas de la Peña Rociera de Rota (SaRR), se celebra desde 1981 y en alguna edición concede premio de nueva letra interpretada sobre Rota (3-1).

- El Concurso Saetas en la Calle, en 2005 (1-0).

- El Concurso de letras de Serranas de Prado del Rey. Concede algunos años premio a mejor nueva letra interpretada (1-0).

\footnotetext{
${ }^{3}$ Por ejemplo: Cante por Peteneras de Paterna de Rivera $=$ PePR.

${ }^{4}$ Más información sobre los premios puede encontrarse en el blog premiosletrasflamencas.blogspot.com donde vamos incluyendo un resumen de la información de los premios de letras inventariados.
} 
- El Concurso de Cante por Soleás, del que encontramos referencia a un año en que se concedía premio de nueva letra pero quedó desierto (0-0).

- El Concurso de Letras Flamencas La Fragua de la Isla (LFLI), que se celebra desde $2014(52-14)^{5}$

- El Concurso de Poesía y Letras Flamencas de Puerto Real (LePR) se celebró en 2017 $(9-1)$.

- El Concurso de letras de saetas de la Peña Buena Gente, que se ha celebrado por primera vez en $2021(3-0)$.

- En Córdoba:

- El Premio Nacional de Letras Flamencas, que organizó el Ateneo de Córdoba entre 1992-1997. Toda la información se puede encontrar en la web del Atened 6 (3-0).

- El Concurso de letras de saetas Ciudad de Lucena (SaLu), desde 1993 (10-1).

- El Premio de letras de Saetas del Certamen de Saetas La Despensa, de Cabra (0-0).

- El Concurso de letras de fandangos de Lucena (FaLu), desde 1998 (15-1).

- El Premio de letras de Pajaronas de Bujalance. Lo organiza la Peña Cultural Flamenca La Pajarona (6-0) $)^{7}$

- En Granada solo tenemos constancia de dos premios de letras antiguos:

- Uno de media granaína, del que aparece una noticia en el periódico Patria de 21 de septiembre de $1940(0-0)$.

- Y otro de 1973 en que se concedió un premio a la mejor letra de exaltación del trabajo (1-0).

- En Huelva tenemos premios de letras de fandangos, en muchos casos concedidos a nuevas letras interpretadas:

- En el Concurso de Fandangos Amparo Correa, de San Silvestre de Guzmán (FaSS), que se celebra desde 1992, se concede un premio a mejor letra alusiva a cualquier aspecto del municipio (4-4).

- El Concurso de letras de fandangos de Villa de Rociana (FaVR) empieza a celebrarse en 2005 (1-4).

- El Concurso de letras de fandangos de Paterna del Campo (FaPC) se celebra desde 2007 (0-1).

- Dentro del Concurso de letras de fandangos Paco Toronjo y Alosno (FaTo) se concede algunos años desde 2007 premios de letras (4-3).

- En el Concurso de Fandangos de Huelva de Santa Olalla del Cala (FaSO), que se celebra desde 2012, se premia la mejor letra alusiva al pueblo o a su patrona o patrón (1-1).

- El Concurso Provincial de Fandangos de Huelva de 2004 (1-0).

- En el Concurso de sevillanas de Villa de Tharsis (SeTh), que se celebra desde 2013, se premia la mejor letra alusiva al pueblo (1-1).

\footnotetext{
${ }^{5}$ Toda la información está en su web: concursodeletras.blogspot.com/

${ }^{6}$ Ver www.ateneodecordoba.com/index.php/Premio_Nacional_de_Letras_Flamencas

${ }^{7}$ La información disponible puede encontrarse en su página web: https://www.lapajarona.es/
} 
(C) Servicio de Publicaciones de la Universidad de Murcia

http://revistas.um.es/flamenco

- Dentro del Concurso de Fandangos de Calañas se ha convocado en 2016 y 2018 premios de letras (5-0).

- En Jaén:

- Un Concurso de mejor letra referente a Pegalajar, en 1969 (0-0).

- Uno de letra de saetas de Radio Jaén (SaRJ) en 1980 (2-1).

- El Concurso de Letras de Tarantas Ciudad de Linares (TaLi), con premio a nueva letra interpretada entre 1983 y 1993, y a nueva letra desde 1994 a 2018 (24-9).

- En Madrid tenemos el Certamen Nacional de Autores de Letras Flamencas (LMad), con una edición en 2000, y puede que otro concurso similar en 2007 (2-1).

- En Málaga:

- En la Porra Flamenca de Archidona hubo premio de letras cuatro años, a un palo distinto por año: seguiriyas, soleás, malagueñas y bulerías (113-0).

- El Premio de Letras Flamencas de Ronda (1970-1973) (32-0).

- El Concurso de letras de verdiales de Málaga, en 1984 y 1985 (2-0).

- El Premio de coplas a la Virgen de Jeva (VeJe). Es un concurso local que se celebra desde 1989 (31-3).

- El Premio de Letras de Saetas de Ronda (SaRo), en 1990 y 1991 (4-2).

- El Premio de Letras Flamencas Mirando a la Torre (LeMT) (1991-2005) (23-9).

- El Concurso de letras flamencas Antonio Fernández Díaz Fosforito (LeFo) del Centro Cordobés de Málaga, entre 1996 y 2001 (3-3).

- El Concurso de Villancicos por Cantes de Málaga (ViMa) (1996-2000) (29-21).

- El Certamen de Versos y Coplas a la Cruz de Mayo, de la Casa de Melilla en Málaga (CoMe), del que tenemos información de 1999 y 2000 (3-3).

- El Concurso de letras flamencas de cantes de Málaga (LeMa), del que conocemos tres ediciones desde 2010 (3-3).

- En Murcia:

- Un Concurso de letras de saetas mineras en La Unión en 1967 (2-0).

- Los premios de letras dentro del Festival del Cante de las Minas de La Unión (FCMU) (1971-2004) (66-3).

- El premio de letras de cartageneras “Ginés Jorquera”, en 2018 y 2021 (4-0).

- En Sevilla:

- El Concurso de saetas de Radio Nacional de España, en 1973 (1-0).

- El Concurso de Letras Francisco Moreno Galván (LFMG), con cinco ediciones, entre 2008 y 2012 (14-2).

- Dentro de la Bienal de Arte Flamenco de Sevilla se concedieron premios de letras únicamente en la primera edición, en 1980 (42-0).

- El Premio de Letras Flamencas Ciudad de Gines (LeCG), del que conocemos que hubo premios en 2008 y 2009 (2-2). 
- El Concurso de Cante Jondo San Marcos - El Saucejo (SMES), que se celebra desde 2012, y algunos años concede premio de letra no registrada (1-1).

- En Vizcaya se convoca desde 1986 en Baracaldo el Premio de la Asociación Andaluza Hijos de Almáchar (AAHA) (163-69).

- Y en general:

- El Premio Poemas del Cante, de Radio Dersa de Tetuán, en 1956 (1-0).

- Se convocó uno de letras de bulerías en Nîmes, Francia, en 2014 (0-0).

- Algunos anunciados por la Junta de Andalucía, de los que únicamente tenemos constancia que se celebró uno de Letras por la Igualdad en 2018 (3-0).

- Canal Sur celebró entre 2018 y 2009 un Concurso de letras de fandangos (FaCS) (2-1).

Vemos que, de los 58 concursos mencionados, en 30 de ellos hubo representación femenina, y el porcentaje de premios concedidos a mujeres ronda el $19 \%$. Hay que tener en cuenta que hemos incluido también letras seleccionadas para publicar, de las que hubo muchas en algunos de los primeros concursos (Certamen de Cante Jondo de Ronda, Porra de Archidona y Bienal de Sevilla), donde todos los letristas premiados y seleccionados fueron hombres, lo que hace que este porcentaje sea muy bajo, pero que, tal como veremos, en años posteriores se modifica al alza este valor.

\section{Premios recibidos por mujeres}

Las tablas que van a continuación incluyen los premios y menciones recibidas por mujeres en los concursos enumerados. Los concursos, identificados por las siglas que les hemos asignado, aparecen por filas, y las letristas premiadas se identifican con abreviaturas que iremos desvelando en los comentarios que incluimos a continuación. En algunos casos se acompaña entre paréntesis un código con el tipo de premio. La figura 1 resume la evolución temporal de las mujeres premiadas y los premios por ellas recibidos. Se muestra la evolución del porcentaje del total acumulado por años de mujeres letristas premiadas respecto al total de premiados (en azul), y del total acumulado de premios recibidos por mujeres respecto al total de premios concedidos (en rojo). Como el considerar el acumulado de todos los años puede lastrar los resultados haciendo que no llegue a verse la evolución real hasta pasados muchos años, hemos incluido también resultados considerando los valores acumulados de los últimos diez años, que se representan con marcas cuadradas, mientras en los acumulados de todos los años las marcas son círculos.

\begin{tabular}{l|c|c|c|c|c|c|c|} 
& 1975 & 1980 & 1988 & 1990 & 1991 & 1993 & 1995 \\
\hline SaRJ & & Vázquez(1) & & & & & \\
\hline TaLi & & & García H. & & García H. & & Blanco \\
\hline SaRo & & & & León(?) & León(?) & & \\
\hline FCMU & Rivero(f) & & & & & & \\
\hline AAHA & & & & & & Lozano(2m) & \\
\hline
\end{tabular}

Tabla 1. Premios y menciones recibidas por mujeres (hasta 1995)

La participación de la mujer como letrista va aumentando lentamente. Vemos que el porcentaje de mujeres premiadas se mantiene al alza en los últimos años, desde el $12.5 \%$ en 2006, y después de haber tenido un aumento importante del $2 \%$ en 1987 hasta el $5 \%$ en 1995, y otro mayor desde ese año hasta el $12 \%$ en 2000. En cuanto a los premios, se llega a un porcentaje de un $20 \%$, que se mantiene estable los últimos 12 años. Si consideramos los acumulados contando los diez 
(C) Servicio de Publicaciones de la Universidad de Murcia

http://revistas.um.es/flamenco

\begin{tabular}{|c|c|c|c|c|c|}
\hline & 1996 & 1997 & 1998 & 1999 & 2000 \\
\hline $\mathrm{MiCa}$ & \multicolumn{5}{|c|}{ Merino } \\
\hline SaLu & \multicolumn{5}{|c|}{ Ortega } \\
\hline TaLi & & González F. & & & \\
\hline LMad & & & & & Aguirre(1) \\
\hline LFMT & Aguirre(3) & Aguirre(3) & & Aguirre(2) & \\
\hline LeFo & Aguirre & Verdú(a) & & & Aguirre \\
\hline \multirow[t]{5}{*}{ ViMa } & Aguirre(1) & Aguirre(2) & Aguirre(3) & Aguirre(2) & Verdú(1) \\
\hline & Jimena(2) & Bugella(3) & Navarro(s) & Fernández M.(s) & Gálvez(s) \\
\hline & Gema V.(s) & Ferrer $(\mathrm{s})$ & Ferrer(s) & Ferrer(s) & $\mathrm{Paz}(\mathrm{s})$ \\
\hline & & & Verdú(s) & Gálvez(s) & Garcés(s) \\
\hline & & & & Atencia(s) & Atencia(s) \\
\hline $\mathrm{CoMe}$ & & & & $\begin{array}{l}\text { Aguirre(2) } \\
\text { Agular(3) }\end{array}$ & Aguirre(3) \\
\hline FCMU & Celdrán(m) & Celdrán(m) & 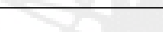 & & \\
\hline$\overline{\mathrm{AAHA}}$ & Aguirre(1emv) & & $\begin{array}{l}\text { Aguirre(1ev) } \\
\text { Verdú(2) } \\
\text { Ramón }(\mathrm{m})\end{array}$ & Verdú(1v) & Aguirre(1emv) \\
\hline
\end{tabular}

Tabla 2. Premios y menciones recibidas por mujeres (de 1996 a 2000)

\begin{tabular}{|c|c|c|c|c|c|}
\hline & 2001 & 2002 & 2003 & 2004 & 2005 \\
\hline LeSL & & Caballero & Merino & & \\
\hline SaRR & & Cordero & & & \\
\hline FaLu & & +2 & & Verdú & 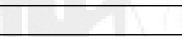 \\
\hline FaSS & & $x^{2}$ & 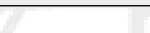 & Vargas & 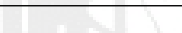 \\
\hline FaVR & & 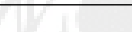 & & & R. Gómez \\
\hline$\overline{\text { LFMT }}$ & & Aguirre(3) & $\begin{array}{c}\text { Ferrer(1) } \\
\text { Bugella(2) }\end{array}$ & Aguirre(2) & Bugella(2) \\
\hline AAHA & Aguirre(2) & Verdú(2v) & $\begin{array}{l}\text { Verdú(1me) } \\
\text { Aguirre }(2 \mathrm{v})\end{array}$ & Verdú(1emv) & Aguirre(1emv) \\
\hline
\end{tabular}

Tabla 3. Premios y menciones recibidas por mujeres (de 2001 a 2005)

\begin{tabular}{|c|c|c|c|c|c|}
\hline & 2006 & 2007 & 2008 & 2009 & 2010 \\
\hline PePR & 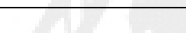 & & & & Gámez \\
\hline FaSS & 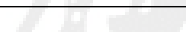 & & & B. Romero & +2 \\
\hline FaVR & +2 & B. Romero & L. Romero & León & 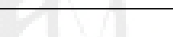 \\
\hline $\mathrm{FaPC}$ & 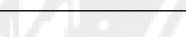 & 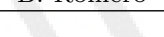 & L. Romero & thens & 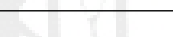 \\
\hline FaTo & 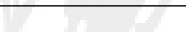 & & Gutiérrez & Gutiérrez & Gutiérrez \\
\hline TaLi & 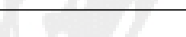 & Sánchez M. & Iglesias & Vega & (n) \\
\hline VeJe & Ruiz & & Lara & res & Arjona \\
\hline LFMG & tents & & Verdú(1) & & García V.(1c) \\
\hline$\overline{\mathrm{LeCG}}$ & $\bar{c}$ & & $\begin{array}{l}\text { Reyes(2) } \\
\text { Antón(3) }\end{array}$ & & +2 \\
\hline$\overline{\mathrm{AAHA}}$ & Bugella(1eom) & Verdú(1mvo) & Gómez R.(3) & Aguirre(1eomvbf) & \\
\hline
\end{tabular}

Tabla 4. Premios y menciones recibidas por mujeres (de 2006 a 2010)

\begin{tabular}{|c|c|c|c|c|c|}
\hline & 2011 & 2012 & 2013 & 2014 & 2015 \\
\hline PePR & & & Granado & & \\
\hline LFLI & & & & $\begin{array}{l}\text { Aguirre(h) } \\
\text { Prats(h) } \\
\text { Gutiérrez(f) }\end{array}$ & $\begin{array}{c}\text { Aguirre(f) } \\
\text { Prats(f) }\end{array}$ \\
\hline FaSS & Rodríguez O. & Lorenzo & & & \\
\hline TaLi & & & N. Romero & & \\
\hline LeMa & & Aguirre(1) & Verdú(1) & & \\
\hline AAHA & & & Verdú(2v) & Verdú(3) & Gómez R.(2) \\
\hline
\end{tabular}

Tabla 5. Premios y menciones recibidas por mujeres (de 2011 a 2015) 
Revista de Investigación sobre Flamenco "La madrugá" n. ${ }^{\circ}$ 19, diciembre 2021, ISSN 1989-6042

\begin{tabular}{|c|c|c|c|c|c|}
\hline & 2016 & 2017 & 2018 & 2019 & 2020 \\
\hline LFLI & Aguirre(f) & $\begin{array}{l}\text { Glez-Amor(f) } \\
\text { Moreno(f) } \\
\text { Prats(f) } \\
\text { Valenciano(f) }\end{array}$ & Valenciano $(\mathrm{f})$ & $\begin{array}{c}\text { Aguirre(1) } \\
\text { Trujillano(f) }\end{array}$ & Aguirre(2) \\
\hline LePR & & Pérez F.(s) & & & \\
\hline $\mathrm{FaSO}$ & Cuesta & & & & \\
\hline TaLi & & & Martos & & \\
\hline SMES & & & Martos & & \\
\hline AAHA & $\begin{array}{l}\text { Verdú(1eomvb) } \\
\text { Lozano }(3)\end{array}$ & & 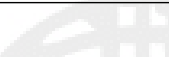 & Verdú(1eomvb) & Aguirre(eov) \\
\hline $\mathrm{FaCS}$ & & & & M. Sánchez(1) & \\
\hline
\end{tabular}

Tabla 6. Premios y menciones recibidas por mujeres (de 2016 a 2020)

años anteriores, vemos que el aumento de mediados de los años noventa produce un incremento mucho mayor en estos valores, llegando hasta un $43 \%$ de premios en 2009, y hasta un porcentaje de letristas del $36 \%$ en 2013. En los últimos años, estos porcentajes a diez años han vuelto a bajar, situándose alrededor del $20 \%$, por lo que parece que la participación femenina tiende a mantenerse a ese nivel, aunque es posible que decaiga rápidamente si la situación sigue siendo como la de este año 2021, en que no encontramos ningún premio recibido por mujeres de entre los catorce contabilizados.

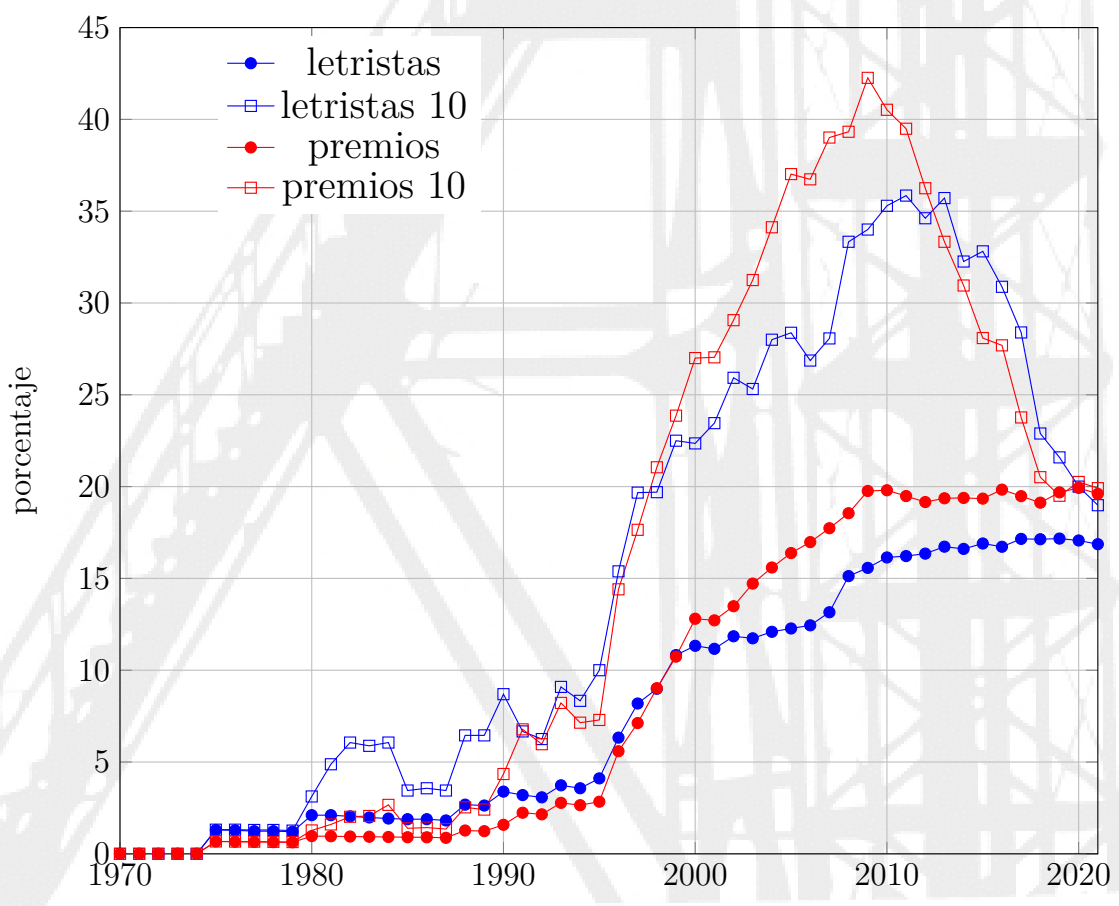

Figura 1. Evolución del porcentaje del total acumulado por años y en los diez últimos años de mujeres letristas y de los premios conseguidos por ellas respecto al total de letristas premiados y de premios concedidos

Comentamos los hitos más importantes en los premios recibidos por mujeres, facilitando una escueta información acerca de cada una de las letristas e incluyendo ocasionalmente algunas de las letras premiadas. 
(C) Servicio de Publicaciones de la Universidad de Murcia

http://revistas.um.es/flamenco

Durante los primeros años, la presencia de la mujer es nula. En 1975 Julia Rivero LópezSerrano (Rivero) recibe una mención como finalista (f) en el Festival del Cante de las Minas, lo que hace aumentar los porcentajes en la figura 1 hasta alrededor del $1 \%$. Este valor es muy bajo debido a que contabilizamos todos los autores seleccionados (todos hombres) en la Porra Flamenca de Archidona y en el Concurso de Cante Jondo de Ronda a principio de los años 70. Vemos en los porcentajes contabilizando los diez años previos que a mitad de los 80 (dejan de contarse los primeros años de los 70) los porcentajes aumentan hasta los 3 y 6 puntos.

Julia Rivero nació en Ciudad Real en 1930, aunque vive en Murcia, donde sigue estando muy activa a los 91 años. Ha publicado numerosos libros de poesía, y su aparición como letrista es ocasional. Esto ocurre con muchas de las letristas que encontramos en este trabajo, que provienen de un contexto literario más general, son cantaoras o aficionadas al flamenco y la literatura. En su caso, la encontramos únicamente como finalista este año, en el que recibió el premio de letras de mineras el conocido José María Pemán, y que fue el único en el que en el Cante de las Minas se mencionan finalistas (Giménez, 2021). En el archivo de La Unión encontramos esta letra señalada por el jurado que, creemos, fue la seleccionada como finalista:

$$
\begin{aligned}
& \text { Echo mi pena a rodar } \\
& \text { cuando canto una minera } \\
& \text { y la pena en mi cantar } \\
& \text { vuelve rodando a mi vera. } \\
& \text { Me dan ganas de llorar. }
\end{aligned}
$$

Sigue un periodo de sequía hasta que en 1980 Josefina Vázquez Florido (Vázquez), poeta aficionada originaria de Jaén, nacida en 1943, obtiene el primer premio en el concurso de letras de saetas organizado por Radio Jaén. Unos años más tarde, en 1988, Francisca García Herrera (García H.) recibe el primero de sus dos premios de letras de tarantas en Linares, de los que no hemos localizado las letras. Ha sido muy activa en la vida flamenca de Linares, siendo presidenta de la Peña Flamenca Femenina Carmen Linares, formó parte del jurado del Concurso de Tarantas en varias ocasiones, y fue profesora de cantaoras, algunas de las cuales ganaron con posterioridad el premio de letras de tarantas.

También en un ámbito local encontramos los premios de Auxiliadora León Marín (León) en el concurso de Saetas de Ronda en 1990 y 1991. Esta autora es otro ejemplo de aficionada, de la que no tenemos ninguna información y solo sabemos por Olea y Tenorio (2010) que recibió premios esos años, pero no conocemos ni qué premios ni las letras.

Ana María Lozano Alcántara (Lozano) es la primera mujer en recibir un premio de la Asociación Andaluza Hijos de Almáchar, tras siete años de estar en marcha este concurso. Solo ha recibido premios en él, el otro en 2016. Por el segundo libro dedicado al concurso (Hijos de Almáchar, 2003) sabemos que nace en Málaga en 1961 y desde muy pequeña reside en Getxo, Vizcaya. La Asociación Andaluza concede tres premios (1, 2 y 3) y varios premios especiales, que empezaron siendo a verdiales (v), aumentando en sucesivas ediciones, llegando a haber premios a seguiriyas (e), soleás (o), malagueñas (m), bulerías (b) y fandangos (f). Incluimos una de sus malagueñas premiadas en 1993:

Dejé yo mis ilusiones

a la orilla de tu amor, y una ola las barrió.

No quieres que te perdone.

No tienes perdón de Dios. 
En el Certamen de Tarantas de Linares, una vez que el premio pasa a ser de nuevas letras (no interpretadas), las letristas premiadas María José Blanco Romero (Romero) y Pilar González Fernández (González F.) provienen de un ámbito literario más general. Por Martínez (2003) sabemos que la primera falleció en 2009, que la segunda es asturiana afincada en Linares, y que las dos eran aficionadas a la literatura, con algunas publicaciones y premios. No hemos encontado las letras premiadas.

Hay un importante aumento en la participación femenina los años 1996 y 1997, que produce un gran incremento en los porcentajes en la figura 1, especialmente en los de los diez años anteriores (los marcados con cuadrados). Seguimos teniendo letristas ocasionales y cuya participación se restringe a su entorno geográfico. Esto pasa con Paloma Celdrán Montoro (Celdrán), que gana el premio de mineras (m) en el Festival del Cante de las Minas de La Unión (su pueblo) en 1996 y 1997. Estudió Filología Hispánica y fue profesora de instituto, recibió algunos premios de poesía y murió muy joven, con 38 años, en Madrid en 2013. Las mineras premiadas estos dos años son, según Sáez y Pérez (2010), la siguientes:

$$
\begin{gathered}
\text { La plata no hace canción, } \\
\text { mirad su fría belleza, } \\
\text { el brillo es rara ilusión, } \\
\text { la verdadera grandeza } \\
\text { es el cante de La Unión. } \\
\text { Sombra y desgarro flamenco. } \\
\text { Suena la copla en la mina } \\
\text { como un profundo lamento. } \\
\text { Minera de plata fina, } \\
\text { vas del corazón al viento. }
\end{gathered}
$$

En el concurso de Villancicos por Cantes de Málaga entre 1996 y 2000 se conceden tres premios (1, 2 y 3$)$ y se seleccionan (s) un total de diez letras que se incluyen cantadas en un CD (no se publicó el de la última edición en 2000). También aquí encontramos algunas letristas restringidas al ámbito geogáfico de Málaga: Concepción Jimena Santaella (Jimena, 2 en 1996); Gema Vega Rodríguez (Gema V., s en 1996); la popular cantante Diana Navarro (Navarro, s 1998), nacida en Huelín, Málaga, en 1978; Sonia Fernández Molina (Fernández M., s 1999), cantaora malagueña; María Lourdes Gálvez del Postigo (Gálvez, s 1999 y 2000), estudiosa del flamenco, con trabajos en prensa y radio, y también cantaora; Mari Carmen Atencia Palma (Atencia, s 1999 y 2000); Gema Garcés Chaves (Garcés, s 2000), que puede que sea una bailaora malagueña; y Ana Paz (Paz, s 2000), que puede ser una compositora y letrista malagueña. Como vemos, la mayoría son letristas ocasionales de las que tenemos poca o ninguna información.

Pero la eclosión de 1996 y 1997 se debe principalmente a Carmen Aguirre Requena (Aguirre), malagueña, y María Victoria Verdú González (Verdú), malagueña de Alhaurín de la Torre. Ellas dos junto con Francisco Acosta Roldán, malagueño de La Calzada pero residente en Alhaurín de la Torre, y Luis Antonio Utrera Madroñero, malagueño, son los cuatro letristas más premiados. Ellos son de una generación algo anterior a la de Carmen y Mariví, estando en los ochenta y pico y ellas sobre los setenta. Pilar Bugella Traver (Bugella), gaditana residente desde niña en Málaga, y Marisa Ferrer Roquero (Ferrer), malagueña, completan el póker de letristas malagueñas que acaparan la mayoría de los premios hasta 2006. Estas cuatro autoras se pueden considerar una "generación" de poetas-letristas, pues coinciden en el tiempo (nacidas entre mitad de los cuarenta y finales de los cincuenta) y en el espacio (malagueñas de origen o de residencia), están ligadas por lazos de amistad (ver foto conjunta en la figura 2 y en algunos 
(C) Servicio de Publicaciones de la Universidad de Murcia

http://revistas.um.es/flamenco

casos de parentesco, son activas en internet 8910 y han realizado obra poética y como letristas recibiendo algunos premios, con Carmen y Mariví algo más orientadas a las letras. Incluimos un par de ejemplos de sus letras premiadas. De Carmen Aguirre unas serranas y seguiriya de María Borrico, del premio Antonio Díaz Fosforito en 2000, que nos proporciona ella misma:

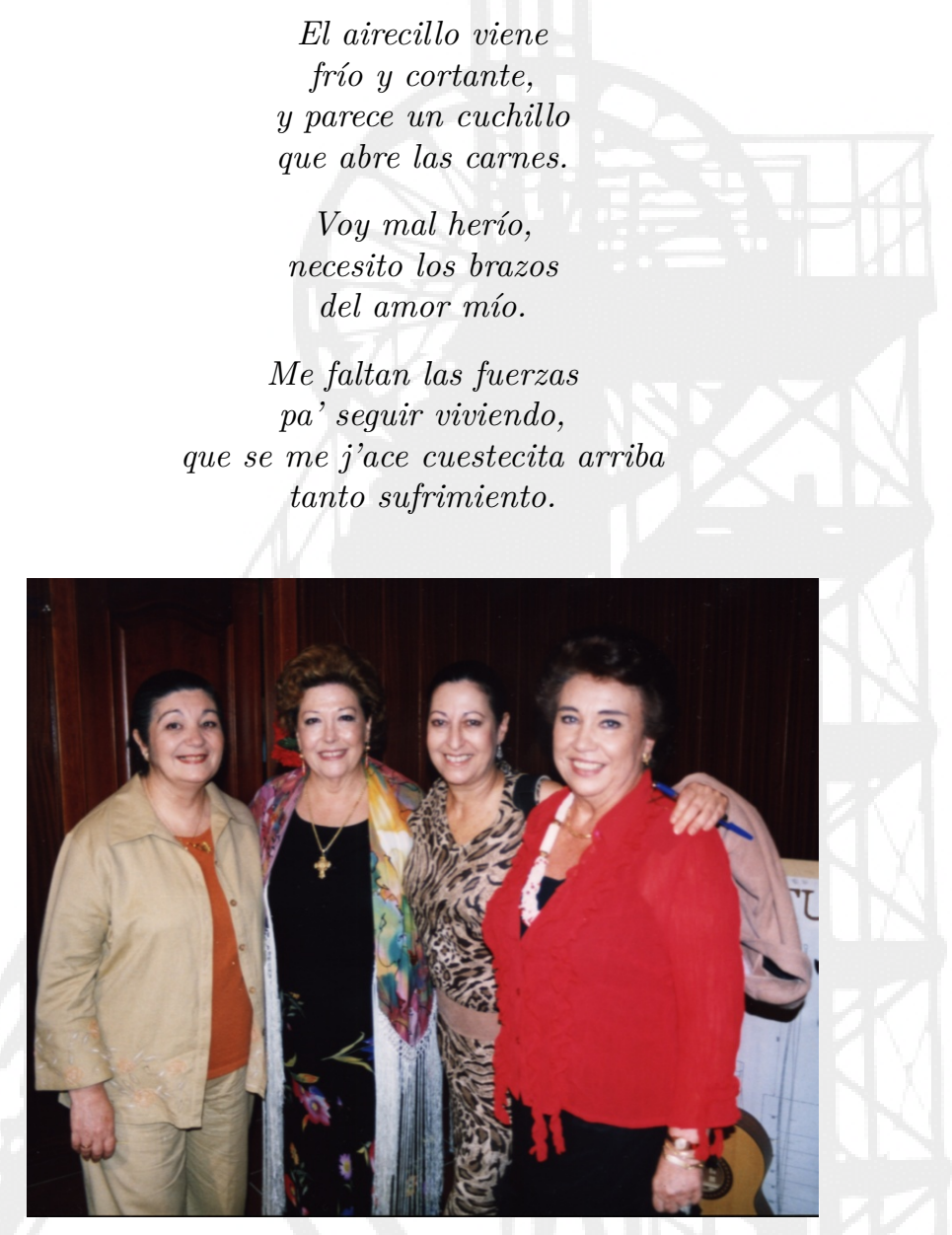

Figura 2. De izquierda a derecha, Pilar Bugella, Mariana Cornejo (cantaora), Carmen Aguirre y Marisa Ferrer, en la recogida de un premio a Pilar y Marisa, en el que Antonio Murciano dio una conferencia y cantó Mariana Cornejo (fotografía facilitada por Carmen Aguirre)

Y de Mariví Verdú unas soleares del primer premio Francisco Moreno Galván en 2008 (Puebla de Cazalla, 2009):

Algo así, pero sin viento

y sin ver la luz del día

tienen que sentir los muertos.

\footnotetext{
${ }^{8}$ Blog de Carmen Aguirre: www.blogger.com/profile/08265901271843689893

${ }^{9}$ Blog de Marisa Ferrer: marisaferrer.blogspot.com/

${ }^{10}$ Blog de Mariví Verdú: dolienteydeoccidente.blogspot.com y garboyflamencura.blogspot.com
} 
Revista de Investigación sobre Flamenco "La madrugá" n. ${ }^{\circ}$ 19, diciembre 2021, ISSN 1989-6042

Mira lo que traigo aqui: una tarde que perdimos $y$ un beso que no te di.

Yo tengo capacidad pa aguantar los temporales... y temporales me dan.

Tiene cosas el amor que nadie puede entender ná más que de dos en dos.

Silencio y silencio es

la pena grande que dice que nunca habrás de volver.

Anoche llovía a mares que el cielo estaba conmigo colmaíto de pesares.

En Mayo tuvo que ser porque es el mes de las flores, de las flores era el mes...

Yo no echo en falta otra cosa que tres vidas que se fueron y que en la tierra reposan.

De tan grande lo que siento sólo sale de mi boca un refilón de lo eterno.

El resto de letristas de finales del siglo XX aparecen ocasionalmente. Encontramos a Virtudes Ortega Jiménez (Ortega), que recibe el premio de letras de saetas de Lucena en 1998; a Teresa Aguilar González (Aguilar), de Casabermeja, Málaga, tercer premio de coplas a la Cruz de Mayo de la Casa de Melilla en Málaga en 1999; y a Juana Ramón Montes (Ramón), de Zaragoza pero residente en Estepona, que recibe el premio de malagueñas de la Asociación Andaluza Hijos de Almáchar en 1998, del que incluimos una de las letras (Hijos de Almáchar, 2003):

Serás sonido de olas, serás campana y jazmín, serás sonido de olas, serás flor en el jardín, ¿malagueña, por qué lloras, será que serás sufrir?

A partir de 1999 aparecen un buen número de letristas, en muchos de los casos cantaoras o con participación en su ámbito local. Su participación suele ser ocasional, lo que produce un importante descenso en el porcentaje de premios recibidos por mujeres a diez años, desde el $43 \%$ en 2009 al $20 \%$ actual. 
(C) Servicio de Publicaciones de la Universidad de Murcia

http://revistas.um.es/flamenco

En Huelva se celebran varios concursos de fandangos y uno de sevillanas, que en algunos casos incluyen premios a mejor nueva letra, y que se conceden al cantaor/cantaora que la interpreta, que en muchos casos no sabemos si es la autora de la letra. Encontramos cantaoras muchas veces muy jóvenes e incluso infantiles: Ángeles Vargas Fernández (Vargas), que recibe el premio de San Silvestre de Guzmán en 2004; Rocío Gómez Moncada la Luvera (R. Gómez), de Almonte, Huelva, que gana el de Villa de Rociana en 2005; Beatriz Romero González (B. Romero), de Palos de la Frontera, Huelva, de 1996, que recibe premios en 2007 en Rociana del Condado y en 2009 en San Silvestre de Guzmán; Lidabeth Romero Pancho (L. Romero), de Almonte, Huelva, premiada en 2008 en Rociana del Condado y en Paterna del Campo; Sonia León (S. León), de Calañas, Huelva, de 1988, premiada en Rociana del Campo en 2009; María Auxiliadora Rodríguez Ortiz (Rodríguez O.), de Moguer, Huelva, con unos 27 años en la actualidad, y premiada en San Silvestre de Guzmán en 2011; Ana Lorenzo Álvez (Lorenzo), de Villanueva de los Castillejos, Huelva, que cuenta en la actualidad unos 21 años, y premiada en San Silvestre de Guzmán en 2012; Carmen Medina Rodríguez (Medina), que recibe el premio de letras de sevillanas en Tharsis en 2015, siendo cantaora infantil con 13 años; y Rocío Belén Cuesta Espina (Cuesta), onubense de 1989, ganadora en Santa Olalla del Cala en 2016.

En Cádiz también hay concursos donde se premian nuevas letras interpretadas, principalmente a principios del presente siglo. Allí encontramos a las cantaoras: María José Merino Vidal Niña de San Judas (Merino), de Sanlúcar de Barrameda, Cádiz, que recibe premios de letras de mirabrás en 1999 y de cantes de Sanlúcar en 2003; Gema Caballero Garzón (Caballero), de Granada de 1979, que gana el de cantes de Sanlúcar en 2002; Manuela Cordero López (Cordero), de Rota, Cádiz, de 1973, aunque ha vivido en varios pueblos de Huelva y Sevilla, que gana el premio de letras de saetas de la Peña Rociera de Rota en 2002; y, años más tarde, África Granado Acuña (Granado), de 21 años y de San José del Valle, Cádiz, premiada por nueva letra de petenera interpretada en el Concurso de Peteneras de Paterna de Rivera en 2013.

En otros concursos también encontramos cantaoras premiadas, pero parece más cierta su autoría de las letras. Reciben premios de letras de tarantas en Linares las cantaoras originarias o residentes en esta ciudad (las letras nos las proporciona la oficina de cultura del ayuntamiento de Linares): Juana Carmona Iglesias (Iglesias), en 2008:

$$
\begin{gathered}
\text { Vagando por los senderos } \\
\text { Entre garganta y garganta... } \\
\text { Vagando por los senderos, } \\
\text { canta tu pena taranta, } \\
\text { que si está vivo el recuerdo } \\
\text { ya no es el minero el que canta. }
\end{gathered}
$$

Belén Vega Rus (Vega), de Linares, 1993, y profesora de cante en el conservatorio de Córdoba, en 2009:

$$
\begin{aligned}
& \text { Linares le dijo al viento, } \\
& \text { escucha bien su quejío, } \\
& \text { Linares le dijo al viento... } \\
& \text { mi taranta es poderío, } \\
& \text { es historia y sentimiento, } \\
& \text { desgarro y escalofrío. }
\end{aligned}
$$

Nazaret Romero López (N. Romero), de Linares (1995), premiada en 2013: 
Revista de Investigación sobre Flamenco "La madrugá" n. ${ }^{\circ}$ 19, diciembre 2021, ISSN 1989-6042

\author{
La tierra tembló esa noche \\ en la mina La Esperanza, \\ la tierra tembló esa noche \\ al escuchar la taranta \\ de ese minero que esconde \\ sus miedos mientras trabaja.
}

Y Alba Martos Garrido (Martos), de Baeza (1994), premiada en 2018, y que también recibe premios de nueva letra interpretada en el Concurso de Cante Jondo de San Marcos-El Saucejo:

$$
\begin{aligned}
& \text { Escucha bien mi taranta } \\
& \text { que la canta el corazón, } \\
& \text { escucha bien mi taranta, } \\
& \text { que la mina marchitó } \\
& \text { la fuerza de mi garganta, } \\
& \text { pero no me derrotó. }
\end{aligned}
$$

Otra cantaora premiada por nueva letra interpretada, y que en este caso sí sabemos que es la autora de la letra, es Virginia Gámez Gil (Gámez), de Málaga (1978), galardonada en Paterna de Rivera en 2010 con las siguientes peteneras que nos proporciona ella misma:

Sus peines son de canela, del cante la emperaora,

de piel morena y trigueña, quiero cantarle a Pastora madre de la petenera.

La niña siempre cantaba "quisiera yo renegar" su voz al cielo clamaba, volver quería a habitar por ver si el mundo cambiaba.

Por otra parte, en concursos con un ámbito local encontramos a: Isabel Gutiérrez Pascual (Gutiérrez), de Soria pero que recibe premios de letras en el Concurso de Fandangos de Alosno, y también la encontramos un año como finalista en el concurso de La Fragua de la Isla; Áurea Sánchez Medina (Sánchez M.), extremeña afincada en Linares, que recibe el premio de letras de tarantas en 2007:

$$
\begin{gathered}
\text { El minero de Linares } \\
\text { creó la dura taranta, } \\
\text { el minero de Linares } \\
\text { quiebra su voz cuando canta, } \\
\text { y el aire de estos lugares } \\
\text { le da fuerza a su garganta. }
\end{gathered}
$$

También se circunscriben a un ámbito local los premios de Marta Reyes González (Reyes) y María Antón Chaves (Antón), segunda y tercera en el Certamen de letras flamencas Ciudad de Gines en 2008. Lo mismo ocurre con la selección para publicación en el certamen de letras de la Peña Canalejas de Puerto Real en 2017 de las soleares de Isabel María Pérez Fernández, muy activa dentro del mundo del flamenco a nivel local y de la provincia de Cádiz, donde ha sido, entre otras cosas, tesorera de la Federación de Peñas Flamencas, y de la que incluimos una soleá: 
(C) Servicio de Publicaciones de la Universidad de Murcia

http://revistas.um.es/flamenco

\author{
Niña, ¿Cuántos volantes \\ tiene tu vestío grana? \\ los mismos que tié de cante \\ la Soleá de Triana.
}

El ejemplo más claro de concurso local es el de coplas a la Virgen de Jeva, que se celebra dentro del encuentro de pandas de verdiales que se realiza en esa ermita, en el Torcal de Antequera en Navidad, y que atrae poetas populares principalmente de zonas montañesas de Málaga. Encontramos tres mujeres que reciben el primer premio: Teresa Ruiz Cantarero (Ruiz), ya fallecida, lo obtiene en 2006; Teresa Lara Jiménez (Lara), de La Higuerica, en el Torcal de Antequera, en 2008; y Francisca Arjona Crespillo (Arjona), poeta aficionada de Antequera, en 2010. De Virgen de Jeva (2008 y 2018) tomamos las coplas premiadas, primero la de 2006 y a continuación las de 2008 y 2010 :

Virgen Purificación,

Virgen de tanta alegría, todo el mundo te venera,

te canta sus poesías

en este Día de Jeva.

Mi tierra tiene dos reinas, dos banderas de amistad, una la Reina de Jeva, la otra la del Torcal, no queremos que se pierdan.

Yo tenía un familiar que en los santos no creía, pero al llegar Navidad a Jeva siempre venía y hasta lo vi de llorar.

En un ámbito más amplio, Canal Sur convocó entre 2018 y 2019 un concurso a mejor fandango en el programa Menuda Noch£ ${ }^{11}$ en el que recibió el primer premio la sevillana María Sánchez, siendo ya nonagenaria:

$$
\begin{gathered}
\text { El fandango es la canción } \\
\text { que nació en Andalucía } \\
\text { el fandango es la canción } \\
\text { donde dolor y alegría } \\
\text { al salir del corazón } \\
\text { se convierte en poesía. }
\end{gathered}
$$

En el Concurso de Letras Francisco Moreno Galván, de Puebla de Cazalla, encontramos en 2010 a Luisa María García Velasco (García V.), que recibe el primer premio por el libro $A$ palos de ciego, trabajo conjunto con José María López Medina. Es una poeta de la que conocemos la obra Anclar en La Alpujarra: un canto a Canjáyar, es originaria de Granada (1967) y reside en Canjáyar (Almería). Del libro premiado en Puebla de Cazalla (2010) incluimos las siguientes alegrías:

${ }^{11}$ Ver www youtube. com/watch?v=ltg4BucLRx0 
Revista de Investigación sobre Flamenco "La madrugá" n. ${ }^{\circ}$ 19, diciembre 2021, ISSN 1989-6042

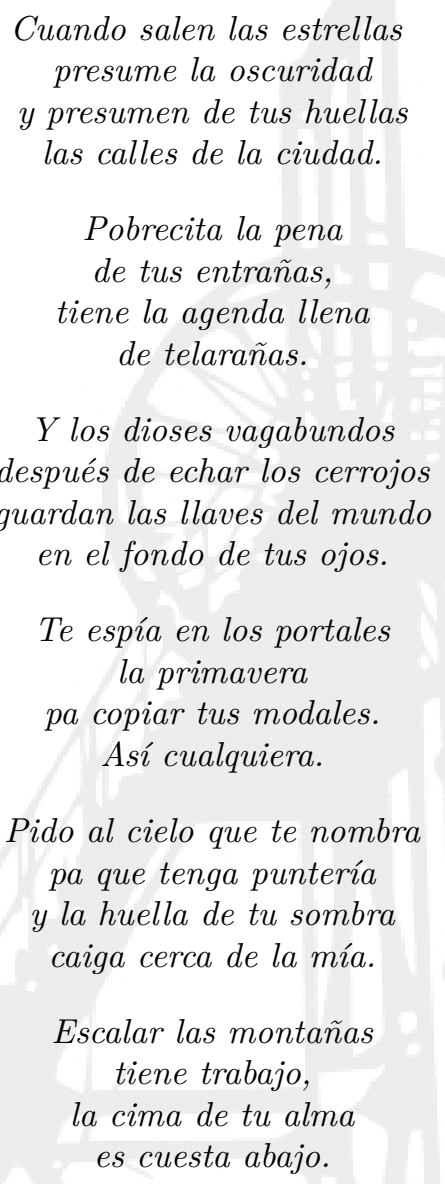

El concurso de la Asociación Andaluza Hijos de Almáchar nos proporciona estos años otra voz femenina, la de la poeta malagueña María Gómez Riera (Gómez R.), que recibe en 2008 el tercer premio y en 2015 el segundo premio, del que incluimos unas alboreás:

\footnotetext{
Mi novia tan blanca como el azahar. Gotas de rubi, rosas de cristal.

Tus primos hermanos están al llegar, que empiece la fiesta vamos a cantar.

Palmas y guitarras han sonado ya y un cantar que dice mañana será.
} 
(C) Servicio de Publicaciones de la Universidad de Murcia

http://revistas.um.es/flamenco

Por último, desde 2014, en el concurso La Fragua de la Isla encontramos una serie de nuevas letristas con menciones de honor (h) o como finalistas (f), lo que nos permite esperar una renovación en la participación femenina en los concursos de letras. Son: María del Mar Prats Yusta (Prats), cordobesa con dos libros de poesía publicados; María del Carmen González-Amor Sánchez (Glez-Amor), sevillana de 1962 que ha publicado artículos sobre flamenco y actúa como cantaora con el nombre de Carmen Amor; María Teresa Moreno Rueda (Moreno), profesora de piano de la Junta de Andalucía e hija de Eustasio Moreno Rodríguez, ganador de dos premios de letras de saetas en Lucena en los noventa; Luz Valenciano Espinar (Valenciano), que creemos es madrileña; y Elena Trujillano Serrano (Trujillano), que suponemos es de Zaragoza. No incluimos letras de estas menciones pues toda la información del concurso, incluidas las letras, se puede encontrar en la web de este concursc 12

En cualquier caso, parece que está costando que se produzca una renovación importante en la participación de mujeres en premios de letras, que permita llegar a las cuotas de participación y premios de la primera década de este siglo, principalmente por las contribuciones de la "escuela de Málaga" y sobre todo de sus dos principales representantes, Carmen Aguirre y Mariví Verdú. En este sentido, ya hemos mencionado la ausencia de mujeres en los premios concedidos en 2021.

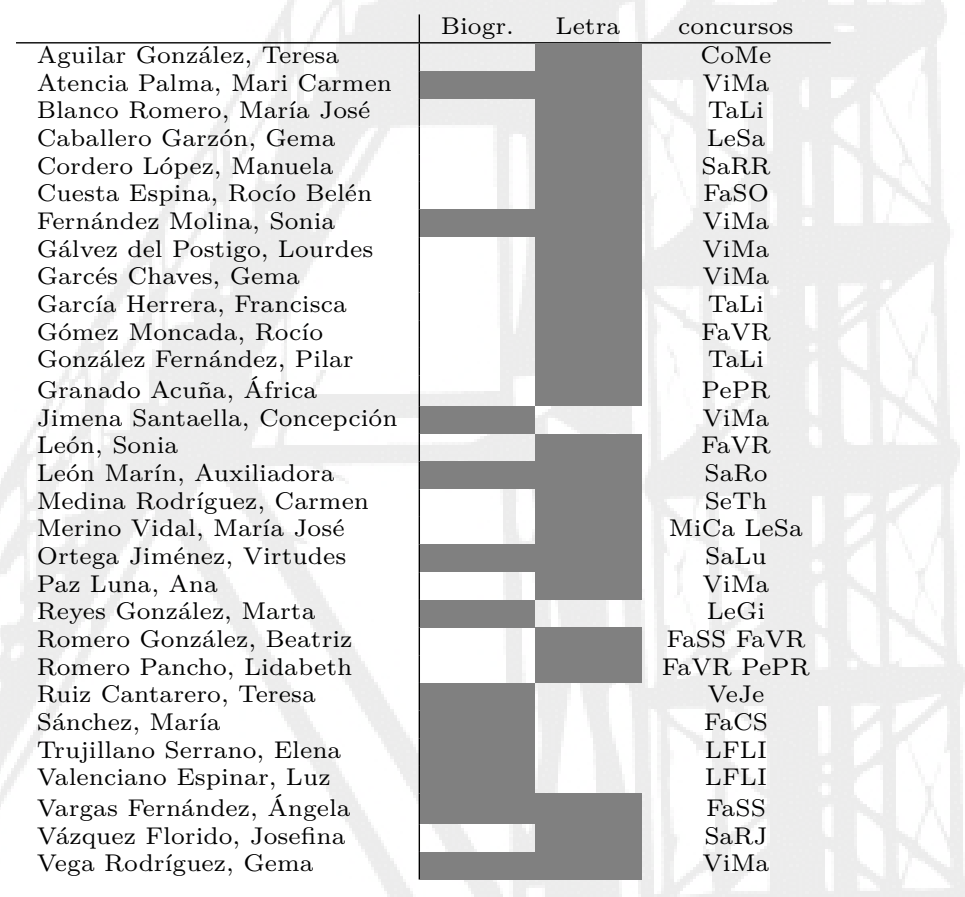

Tabla 7. Datos por completar (en gris) para distintas letristas y fuentes a consultar para la tarea

\section{Conclusiones y trabajo futuro}

Hemos analizado la participación femenina en concursos de letras flamencas, a través de los premios y menciones recibidos en ellos por mujeres. Como en muchos otros campos de la actividad

\footnotetext{
${ }^{12} \operatorname{Ver}$ http://concursodeletras.blogspot.com/
} 
Revista de Investigación sobre Flamenco "La madrugá" n. ${ }^{\circ}$ 19, diciembre 2021, ISSN 1989-6042

cotidiana, la mujer está infrarrepresentada. Lo mismo ocurre en otros apartados del flamenco, a excepción del baile. Apreciamos un aumento de la participación y los premios recibidos los últimos años, estancados en la actualidad entre un 17 y un $20 \%$. Es deseable que la mujer continúe aumentado su cuota de participación en la escritura de letras, y quizás esto contribuiría a una visión más femenina de la letra flamenca. No sabemos si hay una visión femenina diferenciada de la masculina, y merecería la pena hacer un análisis comparativo de la temática de las letras escritas por hombres y por mujeres.

Dentro de nuestro trabajo para inventariar los premios de letras flamencas, querríamos localizar todos los premios convocados y concedidos. Por ahora hemos encontrado 58 premios, y mujeres galardonadas en 30 de ellos. Hay que seguir investigando en esa dirección, pues de algunos eventos no conocemos todos los premios, y puede que haya algún concurso del que no tengamos noticia, por lo que podrían aparecer más mujeres pemiadas. También buscamos alguna información biográfica y bibliográfica de las letristas premiadas, y al menos alguna letra premiada por cada una de ellas. De las letristas que hemos enumerado hay algunas de las que nos falta información. La tabla 7 muestra con casillas en gris los datos que nos faltan por completar de algunas autoras. Del total de 57 letristas, nos faltan datos de tipo biográfico y bibliográfico de 12 (un 21.1\%), y alguna letra premiada de 24 (un 42.1\%). Merece la pena seguir buscando esa información, recurriendo a publicaciones relacionadas con esos concursos, y a las peñas y ayuntamientos que los organizan.

\section{Referencias}

Alarcón SuÁrez, Miguel (2004). La Peña Flamenca Torre del Cante. Alhaurín de la Torre: Concejalía de Cultura del Ayuntamiento de Alhaurín de la Torre.

Giménez CÁnovas, Domingo (2021). Concursos de letras flamencas en el Festival Internacional del Cante de las Minas de La Unión (1971-2004). Murcia: Diego Marín.

Hijos de Almáchar (2003). Letras flamencas II: premiadas en los concursos internacionales convocados por la Asociación Andaluza "Hijos de Almáchar". Málaga: Centro de Ediciones de la Diputación de Málaga.

Martínez Aguilar, Lorenzo (2003). El grupo literario "Amanecer" de Linares: un colectivo heterogéneo de voces y palabras. Siete Esquinas, n. ${ }^{\circ}$ 5, pp. 5-90. Recuperado de dialnet.unirioja.es/ servlet/articulo?codigo $=4511069$.

Olea Barbarán, Gabriel y Tenorio González, María de la Paz (2010). Flamenco en Ronda y la serranía. Ronada: La Serranía.

Puebla de CAzalla (2009). Letras flamencas: publicación de los trabajos ganadores del I concurso de letras flamencas "Francisco Moreno Galván". La Puebla de Cazalla: Concejalía de Cultura Ayuntamiento de La Puebla de Cazalla.

Puebla de CAzAlla (2010). Letras flamencas III: publicación de los trabajos ganadores del III concurso de letras flamencas "Francisco Moreno Galván". La Puebla de Cazalla: Concejalía de Cultura Ayuntamiento de La Puebla de Cazalla.

SÁez, Asensio y PÉrez, José Alfonso (2010). Crónicas del Festival Internacional del Cante de las Minas. Torrepacheco (Murcia): Ayuntamiento de La Unión.

Virgen de Jeva (2008). Segunda década de la recuperación del tradicional día de Jeva. Antequera: Hermandad Virgen de Jeva.

ViRgen DE Jeva (2018). Tercera década de la recuperación del tradicional día de Jeva. Antequera: Hermandad Virgen de Jeva. 